\title{
Alfred Marshall's cardinal theory of value: the strong law of demand
}

\author{
Donald J. Brown · Caterina Calsamiglia
}

Received: 29 November 2013 / Accepted: 8 January 2014 / Published online: 22 January 2014 (C) SAET 2014

\begin{abstract}
We show that all the fundamental properties of competitive equilibrium in Marshall's cardinal theory of value, as presented in Note XXI of the mathematical appendix to his Principles of Economics (1890), derive from the Strong Law of Demand. That is, existence, uniqueness, optimality, and global stability of equilibrium prices with respect to tatonnement price adjustment follow from the cyclical monotonicity of the market demand function in the Marshallian general equilibrium model. We propose a refutable model of Marshall's cardinal theory of value: the Marshallian equilibrium inequalities. We show that the Marshallian equilibrium inequalities have a solution iff the finite market data set consisting of observations on market prices and social endowments is cyclically monotone.
\end{abstract}

Keywords Cardinal utility - Quasilinear utility · Cyclical monotonicity

JEL Classification $\quad \mathrm{B} 13 \cdot \mathrm{D} 11 \cdot \mathrm{D} 51$

\section{Introduction}

We show that all the fundamental properties of competitive equilibrium in Marshall's cardinal theory of value, as presented in Note XXI of the mathematical appendix to his Principles of Economics (1890), derive from the Strong Law of Demand. That

We found the comments and suggestions of the Associate Editor extremely helpful.

\footnotetext{
D. J. Brown $(\varangle)$

Yale University, New Haven, USA

e-mail: donald.brown@yale.edu

C. Calsamiglia

Universitat Autonoma de Barcelona, Barcelona, Spain
} 
is, existence, uniqueness, optimality, and global stability of equilibrium prices with respect to tatonnement price adjustment follow from the cyclical monotonicity of the market demand function in the Marshallian general equilibrium model.

Marshall in Note XXI of the mathematical appendix to his Principles of Economics (1890) presents a fully articulated theory of general equilibrium in market economies. This is not the partial equilibrium model with only two goods usually associated with Cournot (1838), Dupuit (1844) or Marshall (1890), nor is it the partial equilibrium model exposited in the first chapter of Arrow and Hahn (1971), or in Chap. 10 of MasColell, Whinston, and Green (MWG) (1995). Marshall's general equilibrium model differs in several essential respects from the general equilibrium model of Walras (1900). In Marshall's model there are no explicit budget constraints for consumers, the marginal utilities of incomes are exogenous constants and market prices are not normalized. He " proves" the existence of market clearing prices, as does Walras, by counting the number of equations and unknowns. Marshall's first order conditions for consumer satisfaction require the gradient of the consumer's utility function to equal the vector of market prices.

A recent modern exposition of the fundamental properties of Marshall's general equilibrium model in Note XXI can be found in Sects. 8.4-8.6 of Bewley (2007), where he calls it "short-run equilibrium". As in Marshall, there are no explicit budget constraints for consumers, the marginal utilities of incomes are exogenous constants and market prices are not normalized. Consumers in Bewley's model satisfy Marshall's first order conditions in a short-run equilibrium. Bewley proves that: (i) a unique shortrun equilibrium exists; (ii) welfare in a short-run equilibrium can be computed using the consumer surplus of a representative agent and (iii) the short-run equilibrium is globally stable under tatonnement price adjustment.

We show that the fundamental properties of competitive equilibrium in Marshall's theory of value as derived in Bewley are immediate consequences of the market demand function satisfying the Strong Law of Demand, introduced by Brown and Calsamiglia (2007). A demand function is said to satisfy the Strong Law of Demand if it is a cyclically monotone function of market prices. Cyclically monotone demand functions not only have downward sloping demand curves, in the sense that they are monotone functions of market prices, but also their line integrals are path-independent and hence provide an exact measure of the change in consumer's welfare in terms of consumer's surplus for a given multidimensional change in market prices. This is an immediate consequence of Roy's identity applied to the indirect utility function for quasilinear utilities, where the marginal utility of income is one. Following Quah (2000), we show that the Strong Law of Demand is preserved under aggregation across consumers. Hence the area under the market demand curve is an exact measure of the change in aggregate consumer welfare for a given multidimensional change in market prices.

Brown and Calsamiglia prove that a consumer's demand function satisfies the Strong Law of Demand, iff the consumer behaves as if she were maximizing a quasilinear utility function subject to a budget constraint. The defining cardinal property of quasilinear utilities, say for two goods, is that the indifference curves are parallel. Consequently, quasilinear utility is measured on an interval scale. It is in this sense that Marshall's general equilibrium model is a cardinal theory of value, where differences 
in a consumer's quasilinear utility levels are a proxy for the consumer's intensity of preferences. The assumption of maximizing a quasilinear utility function subject to a budget constraint is made by MWG in their discussion of partial equilibrium analysis in the two goods case, but there is no explicit mention of the Strong Law of Demand in their analysis. In Bewley's discussion of short-run equilibrium, there is no explicit mention of the Strong Law of Demand or maximizing a quasilinear utility function subject to a budget constraint.

The fundamental difference between the Marshallian and Walrasian theories of value is the measurement scale for utility levels of consumers. In the Marshallian model the measurement scale is cardinal, more precisely an interval scale, where the family of indifference curves is a metric space isometric to the positive real line. That is, fix any open interval $I \equiv(\bar{x}, r): r>0\} \subset R_{++}^{2}$ and assume that the quasilinear utility function $U(x, y)=v(x)+y$ on $\mathbb{R}_{++}^{2}$ is smooth, monotone and strictly concave. If $(\bar{x}, \bar{y}) \in I$ then define

$$
\Phi(\bar{y}) \equiv\left\{(x, y) \in \mathbb{R}_{++}^{2}: U(x, y)=U(\bar{x}, \bar{y})\right\}
$$

i.e., the unique indifference curve of $U(x, y)$ passing through $(\bar{x}, \bar{y})$. $\Phi$ is a one-to-one map from the metric space $I$ onto $\Gamma[U]$, the family of indifference curves for $U$. As such, $\Phi$ induces a metric on $\Gamma[U]$, where if $(\alpha, \beta) \in \Gamma[U] \times \Gamma[U]$, then

$$
\operatorname{dist}(\alpha, \beta) \equiv \mid\left(\Phi^{-1}(\alpha)-\Phi^{-1}(\beta) \mid\right.
$$

That is, $\Phi^{-1}$ is an isometric imbedding of $\Gamma[U]$ into $\mathbb{R}_{++}$. Of course, this metric representation extends to quasilinear utilities on $\mathbb{R}_{++}^{N+1}$ of the form

$$
U(x, y)=v(x)+y \quad \text { where } x \in \mathbb{R}_{++}^{N} \text { and } y \in \mathbb{R}_{++}
$$

That is, $\Phi^{-1}$ is an isometric imbedding of $\Gamma[U]$ into $\mathbb{R}_{++}$

In the Walrasian model, the measurement scale for utility levels is an ordinal scale, where only properties of consumer demand derivable from indifference curves are admissible in the Walrasian model, e.g., the marginal utility of income is not an admissible property. Ordinal scales are sufficient for characterizing exchange efficiency in terms of Pareto optimality or compensating variation or equivalent variation. Unfortunately, a meaningful discussion of distributive equity requires interpersonal comparisons of aggregate consumer welfare. If there is a representative agent endowed with a quasilinear utility function, then the equity of interpersonal changes in aggregate consumer welfare is reduced to intrapersonal changes in the consumer surplus of the representative agent. Hence notions of distributional equity are well defined and exact in the Marshallian cardinal theory of value.

We argue that rationalizing consumer demand with quasilinear cardinal utility functions is comparable to rationalizing consumer demand with neoclassical ordinal utility functions. In the latter case Afriat (1967) proved that neoclassical rationalization is refutable and in the former case, Brown and Calsamiglia extended his analysis to show that quasilinear rationalization is also refutable. Hence in both cases, the debate about 
the efficacy of either the cardinal or ordinal model of utility maximization subject to a budget constraint has been reduced to an empirical question that is resolvable in polynomial time using market data and interior point methods.

Brown and Matzkin (1996) extended the notion of rationalizing the consumer's demand data as utility maximization subject to a budget constraint to rationalizing market data with market economies. They define a Walrasian pure exchange economy, where consumers are endowed with smooth, monotone concave utility functions and incomes, as rationalizing a finite set of observations of market prices, income distributions and social endowments if the observed data lie on the equilibrium manifold defined by the excess market demand function of the given exchange economy. As is well known, the Afriat inequalities constitute necessary and sufficient conditions to rationalize the demand data of a consumer as utility maximization subject to a budget constraint. The Walrasian equilibrium inequalities, introduced by Brown and Matzkin, is a family of multivariate polynomial inequalities consisting of the Afriat inequalities for each consumer, the budget constraints of each consumer in each observation and the market clearing equations in each observation. The parameters in the Walrasian equilibrium inequalities are the market prices, the income distributions and the social endowment in each observation. The unknowns are the utility levels, the marginal utilities of income and the individual demands of consumers in each observation. As such, this is a non-convex family of inequalities. Brown and Matzkin show that the observed market data are rationalized by some Walrasian pure exchange economy iff the Walrasian equilibrium inequalities are solvable for the given market data set.

In the third section of the paper, we propose the Marshallian equilibrium inequalities and derive the refutable implications of Marshall's cardinal theory of value. That is, a finite family of multivariate polynomial inequalities, the Afriat inequalities for quasilinear utilities derived by Brown and Calsamiglia, and the market clearing equations in each observation. Here the parameters are the observed market prices and social endowments in each observation and the unknowns are the unobserved utility levels and unobserved demands of individual consumers in each observation. As a corollary, this result implies that Bewley's notion of short-run equilibrium is also refutable.

A consequence of the Tarski-Seidenberg Theorem is the existence of a finite family of multivariate polynomial inequalities - the revealed Marshallian equilibrium inequalities - where the unknowns are the parameters of the Marshallian equilibrium inequalities such that, for a given market data set, the Marshallian equilibrium inequalities are solvable for the utility levels and demands of consumers in each observation iff the market data set solves the revealed Marshallian equilibrium inequalities. This consequence of the Tarski-Seidenberg theorem is a deep and remarkable extension of the well-known fact that the quadratic equation:

$$
a x^{2}+b x+c=0
$$

with real coefficients $a, b$ and $c$ has real solutions iff the resultant

$$
\left(b^{2}-4 a c\right) \geq 0
$$


For economists, a more interesting consequence of the Tarski-Seidenberg Theorem is Varian (1983) Theorem: the Afriat inequalities are solvable for the consumer's utility levels and marginal utilities of income in each observation for a given data set consisting of a finite family of observations on market prices and the consumer's demands, iff the market data satisfies GARP iff the demand data can be rationalized by a non-satiated, concave utility function

In Bewley (1980) characterization of the short-run equilibrium model as a representative agent model-see also Sects. 8.5 and 8.6 of his (2007) monograph, he defines a representative agent as the Marshallian social welfare function of the form

$$
\begin{gathered}
W(e) \equiv \max _{\left\{x_{1}, \ldots, x_{n I}\right\} \in \mathbb{R}_{++}^{n I}}\left[\sum_{i=1}^{I} \frac{1}{\lambda_{i}} g_{i}\left(x_{i}\right)\right] \\
\text { s.t. } \sum_{i=1}^{I} x_{i}=e .
\end{gathered}
$$

where consumers are endowed with smooth, monotone utility functions $g_{i}(x)$ and constant marginal utilities of income $\lambda_{i}$ and $e$ is the social endowment. Brown and Calsamiglia have shown that the assumption of constant marginal utility of income is equivalent to assuming that

$$
U(x) \equiv \frac{1}{\lambda_{i}} g_{i}(x)
$$

is quasilinear. The representative agent is said to rationalize the market data if she is endowed with the social welfare function $W(e)$ where the social endowment in each observation is her utility maximizing demand subject to the budget constraint defined by the market prices and the social endowment. That is,

$$
W(e)=\max _{p \cdot y \leq p \cdot e} W(y)
$$

We show the Marshallian equilibrium inequalities are solvable for the utility levels and demands of consumers in each period for a given data set consisting of a finite family of observations on market prices and social endowments iff the market data is cyclically monotone iff there exists a representative agent endowed with a quasilinear social welfare function that rationalizes the observed market data. As such, cyclical monotonicity of the observed market data exhausts the refutable implications of Marshall's cardinal theory of value and constitutes the revealed Marshallian equilibrium inequalities. There is no comparable result for the Walrasian theory of value.

For ease of exposition we limit our discussion to pure exchange economies but, as suggested by the analysis of short-run equilibrium in Bewley, all of our results extend to Marshall's general equilibrium model with production. 


\section{A cardinal theory of value}

For completeness, we recall Afriat's seminal (1967) theorem on rationalizing consumer demand data $\left(p_{r}, x_{r}\right), r=1,2, \ldots, N$, with an ordinal utility function and the Brown and Calsamiglia (2007) extension of Afriat's theorem to rationalizing consumer demand data with a cardinal utility function, i.e., a quasilinear utility function. Afriat showed that the finite set of observations of market prices and consumer demands at those prices can be rationalized by an ordinal utility function iff there exists a concave, continuous, non-satiated utility function that rationalizes the data. That is, there exists a concave, continuous, non-satiated utility function $U$, such that for $r=1,2, \ldots, N$ :

$$
U\left(x_{r}\right)=\max _{p_{r} \cdot x \leq p_{r} \cdot x_{r}} U(x) .
$$

Moreover, this rationalization is equivalent to two other conditions: (1) The "Afriat inequalities" :

$$
U_{j} \leq U_{k}+\lambda_{k} p_{k} \cdot\left(x_{j}-x_{k}\right) \text { for } j, k=1,2, \ldots, N
$$

are solvable for utility levels $U_{r}$ and marginal utilities of income $\lambda_{r}$ and (2) the data satisfies cyclical consistency, a combinatorial condition that generalizes the strong law of revealed preference to allow thick indifference curves. See Varian (1983) for proofs. Brown and Calsamiglia showed that the data can be rationalized by a quasilinear utility function iff the Afriat inequalities have a solution where the $\lambda_{r}=1$; that is, constant marginal utility of income. Moreover, they show that quasilinear rationalization is equivalent to another combinatorial condition on the data, cyclical monotonicity. Rockafellar (1970) introduced the notion of cyclical monotonicity as a means of characterizing the subgradient correspondence of a convex function. For smooth strictly concave functions $f$ the gradient map $\partial f(x)$ is cyclically monotone if for all finite sequences $\left(p_{t}, x_{t}\right)_{t=1}^{T}$, where $p_{t}=\partial f\left(x_{t}\right)$ :

$$
x_{1} \cdot\left(p_{2}-p_{1}\right)+x_{2} \cdot\left(p_{3}-p_{3}\right)+\cdots+x_{T} \cdot\left(p_{1}-p_{T}\right) \geq 0 .
$$

Hildenbrand (1983) extension of the law of demand to multicommodity market demand functions requires the demand function to be monotone. He showed that it is monotone if the income distribution is price independent and has downward sloping density. Subsequently, Quah (2000) extended Hildenbrand's analysis to individual's demand functions. His sufficient condition for monotone individual demand is in terms of the income elasticity of the marginal utility of income. Assuming that the commodity space is $\mathbb{R}_{++}^{n}$, we denote the demand function at prices $p \in \mathbb{R}_{++}^{n}$ by $x(p)$. This demand function satisfies the law of demand or is monotone if for any pair $p, p^{\prime} \in \mathbb{R}_{++}^{n}$ of prices

$$
\left(p-p^{\prime}\right) \cdot\left[x(p)-x\left(p^{\prime}\right)\right]<0 .
$$

This means, in particular, that the demand curve of any good is downward sloping with respect to its own price, i.e., satisfies the law of demand if all other prices are 
held constant. We denote the Marshallian consumer optimization problem by $(M)$ :

$$
\max _{x_{i} \in \mathbb{R}_{++}^{n}} \frac{1}{\lambda_{i}} g_{i}\left(x_{i}\right)-p \cdot x_{i}
$$

where $g_{i}$ is a smooth, strictly increasing and strictly concave utility function on $\mathbb{R}_{++}^{n}$, $\lambda_{i}$ is the exogenous marginal utility of income, $p$ is the vector of market prices and $x_{i}$ is the consumption bundle. In this model there are no budget constraints and prices are not normalized. This specification of the consumer's optimization problem rationalizes the family of equations defining Marshall's general equilibrium model (absent production) in his Note XXI.

Brown and Calsamiglia show that

Theorem $1(M)$ is equivalent to the consumer quasilinear optimization problem $(Q)$ :

$$
\max _{\text {s.t. } p \cdot x_{i}+y=I} U_{i}\left(x_{i}\right)+y, \quad \text { where } x_{i} \in R_{++}^{n}
$$

where

$$
U_{i}\left(x_{i}\right) \equiv \frac{1}{\lambda_{i}} g_{i}\left(x_{i}\right)
$$

Proof They have the same first order conditions, hence strict concavity of $g_{i}\left(x_{i}\right)$ guarantees that they have the same unique solution.

We define a Marshallian general equilibrium model as an exchange economy with a social endowment and a finite number of consumers endowed with smooth, strictly increasing and strictly concave utility function and a fixed exogenous marginal utility of income. This is the general equilibrium model explicitly used by Bewley in his discussion of short-run equilibria and implicitly used by Marshall in his Note XXI. It follows from Theorem 1 that an equivalent formulation of the Marshallian general equilibrium model is an exchange economy with a social endowment and a finite number of consumers endowed with smooth, strictly increasing and strictly concave quasilinear utility functions. In the next theorem, we derive the fundamental the market demand function of the Marshallian general equilibrium model.

Theorem 2 If there are I consumers, where consumer $i$ 's optimization problem is given by $(M)$, then the market demand function satisfies the Strong Law of Demand.

Proof Let $h_{i}(p)=\frac{1}{\lambda_{i}} g_{i}\left(x_{i}(p)\right)-p \cdot x_{i}(p)$ be the optimal value function for $(M)$ for consumer $i$. Applying the envelope theorem we know that $\partial h_{i}(p)=-x_{i}(p)$. Let $H(p)=\sum_{i=1}^{I} h_{i}(p)$, then $\partial H(p)=\sum_{i=1}^{I} \partial h_{i}(p)=\sum_{i=1}^{I}-x_{i}(p)$. Therefore the market demand at prices $p$ is $X(p)=\sum_{i=1}^{I} x_{i}(p)=-\sum_{i=1}^{I} \partial h_{i}(p)=-\partial H(p)$. Since $-h_{i}(p)$ is a concave function, $-\partial h_{i}(p)$ and $-\partial H(p)$ are cyclically monotonesee Theorem 24.8 in Rockafellar (1970). Hence, the market demand function $X(p)$ satisfies the Strong Law of Demand. 
Corollary 3 The Marshallian general equilibrium model has a unique equilibrium price vector that is globally stable under tatonnement price adjustment.

Proof Every cyclically monotone map is a monotone map. That is, market demand functions satisfying the Strong Law of Demand a fortiori satisfy the Law of Demand. Hildenbrand (1983) shows that economies satisfying the Law of Demand have a unique equilibrium price vectors that are globally stable under tatonnement price adjustment.

Theorem 4 The welfare of the representative agent in the Marshallian general equilibrium model, can be computed using consumer surplus.

Proof The representative agent's utility function in Bewley's Marshallian general equilibrium model is given by the following social welfare function:

$$
\begin{gathered}
W(e)=\max _{\left\{x_{1}, \ldots, x_{I}\right\} \in \mathbb{R}_{++}^{n I}}\left[\sum_{i=1}^{I} \frac{1}{\lambda_{i}} g_{i}\left(x_{i}\right)\right] \\
\text { s.t. } \sum_{i=1}^{I} x_{i}=e .
\end{gathered}
$$

Bewley shows that $(\bar{p}, x(\bar{p}))$ is an equilibrium of the exchange economy with consumers $\left\{\left(g_{i}, \lambda_{i}\right)\right\}_{i=1}^{I}$ and social endowment $\bar{e}$ iff

$$
\bar{e}=\arg \max _{e \in R_{++}^{N}}\{W(e)-\bar{p} e\}
$$

Hence $(\bar{p}, x(\bar{p}))$, the market demand function, is the demand function of the representative agent. Brown and Calsamiglia show that if the consumer's demand function is cyclically monotone then her welfare can be computed using consumer surplus. Hence it follows from Theorem 2 that the welfare of the representative agent can be computed using consumer surplus.

Theorem 5 The equilibrium map, $p(e)$, in Marshall's general equilibrium model is cyclically monotone in $e$, the social endowment.

Proof Bewley shows that $(\bar{p}, x(\bar{p}))$ is an equilibrium of the exchange economy with consumers $\left\{\left(g_{i}, \lambda_{i}\right)\right\}_{i=1}^{I}$ and social endowment $\bar{e}$ iff

$$
\bar{e}=\arg \max _{e \in R_{++}^{N}}\{W(e)-\bar{p} e\} .
$$

where,

$$
\begin{gathered}
W(e)=\max _{\left\{x_{1}, \ldots, x_{I}\right\} \in \mathbb{R}_{++}^{n I}}\left[\sum_{i=1}^{I} \frac{1}{\lambda_{i}} g_{i}\left(x_{i}\right)\right] \\
\text { s.t. } \sum_{i=1}^{I} x_{i}=e .
\end{gathered}
$$


Equivalently, for a given $\bar{e}$, the price vector $\bar{p}$ such that $\bar{e}=\arg \max _{e \in R_{++}^{N}}\{W(e)-$ $\bar{p} e\}$ will be the unique competitive equilibrium price vector for this exchange economy. Let $H(\bar{p})=\max _{e \in \mathbb{R}_{++}^{I}}\{W(e)-\bar{p} \cdot e\}$, then it follows that

$$
H(\bar{p}) \equiv \sum_{t=1}^{T} h_{t}(\bar{p})
$$

if $\bar{p}$ is a competitive equilibrium vector of prices. Hence,

$$
-\left.(\partial H / \partial p)\right|_{\bar{p}}=\sum_{t=1}^{T}-\left.(\partial H / \partial p)\right|_{\bar{p}}=\sum_{t=1}^{T} x_{t}(\bar{p})=x(\bar{p})=e .
$$

The equilibrium map $p(e)$ is again the inverse of the demand function of the representative consumer. From (Rockafellar (1970), p. 219), Corollary 23.5.1 we know that if $g$ is a continuous concave function on $\mathbb{R}_{++}^{I}$ then $p \in \partial g(x)$ iff $x \in-\partial h(p)$. It follows from this duality relationship that $\bar{p}$ is the unique equilibrium price vector for the social endowment $\bar{e}$ if and only if $\bar{p}=\left.(\partial W / \partial e)\right|_{e=\bar{e}}$ and $-\left.(\partial H / \partial p)\right|_{\bar{p}}=\bar{e}$. Because $g_{i}$ is strictly concave, $W(e)$ is strictly concave as well. By Theorem 24.8 in Rockafellar (1970) we know that the gradient map of a concave function is cyclically monotone, which implies that the gradient map $\left.\vec{e} \rightarrow\left(\frac{\partial W}{\partial e}\right)\right|_{e=\bar{e}}=\bar{p}$ is cyclically monotone.

\section{The Marshallian equilibrium inequalities}

Brown and Matzkin (1996) define the Walrasian equilibrium inequalities for an exchange economy as a finite family of multivariate polynomial inequalities, consisting of the Afriat inequalities for each consumer, the budget constraints of each consumer and the market clearing equations in each observation. The unknowns in the equilibrium inequalities are the unobserved demands of individual consumers and unobservable theoretical constructs such as utility levels and marginal utilities of income. The parameters in the equilibrium inequalities are observable market data such as market prices, social endowments and the income distributions. Brown and Matzkin define the equilibrium inequalities as refutable if there exists a finite family of multivariate polynomial inequalities in the parameters - the revealed Walrasian equilibrium axiom - such that the Walrasian equilibrium inequalities are solvable iff the observed market data satisfies the revealed Walrasian equilibrium inequalities. They show that the Walrasian model is refutable iff there exists a data set where the Walrasian equilibrium inequalities are consistent, i.e., solvable and a second data set where the Walrasian equilibrium inequalities are falsifiable, i.e., unsolvable. This is a non-constructive existence proof. Brown and Matzkin do not derive the revealed Walrasian equilibrium inequalities. In principle the revealed Walrasian equilibrium inequalities can be derived using quantifier elimination, as proposed by Tarski and Seidenberg. This is a constructive existence proof, but unfortunately this method is 
highly inefficient—see Basu (2011) survey on "Algorithms in Real Algebraic Geometry".

We propose a refutable model of Marshall's cardinal theory of value. That is, a finite family of multivariate polynomial inequalities, consisting of the Afriat inequalities for quasilinear utilities derived by Brown and Calsamiglia, and the market clearing equations in each observation, where the parameters are the observed market prices and social endowments in each observation and the unknowns are the unobserved utility levels and unobserved demands of individual consumers in each observation. Using Bewley (1980) characterization of the short-run equilibrium model as a representative agent model-also see Sects. 8.5 and 8.6 of his (2007) monograph, we derive the revealed Marshallian equilibrium inequalities.

A representative agent is said to rationalize the market data if she is endowed with a utility function, where the social endowment in each observation is her utility maximizing demand subject to the budget constraint defined by the market prices and the social endowment. We show that there exists a representative agent endowed with a quasilinear utility function that rationalizes the market data, consisting of observed pairs of market prices and social endowments, iff the observed pairs of market prices and social endowments are cyclically monotone.

Theorem 6 There exists a representative agent endowed with a quasilinear utility function that rationalizes the market data, consisting of observed pairs of market prices and social endowments, iff the observed pairs of market prices and social endowments are cyclically monotone.

Proof Bewley proves that $(\bar{p}, x(\bar{p}))$, is the observed market demand of the representative agent, where $x(\bar{p})=\bar{e}$ is an equilibrium of the exchange economy with consumers endowed with $\left\{\left(g_{i}, \lambda_{i}\right)\right\}_{i=1}^{I}$ and social endowment $\bar{e}$

$$
\begin{gathered}
W(e)=\max _{\left\{x_{1}, \ldots, x_{I}\right\} \in \mathbb{R}_{++}^{n I}}\left[\sum_{i=1}^{I} \frac{1}{\lambda_{i}} g_{i}\left(x_{i}\right)\right] \\
\text { s.t. } \sum_{i=1}^{I} x_{i}=e .
\end{gathered}
$$

Bewley shows that $(\bar{p}, x(\bar{p}))$ is an equilibrium of the exchange economy with consumers endowed with $\left\{\left(g_{i}, \lambda_{i}\right)\right\}_{i=1}^{I}$ and social endowment $\bar{e}$ iff

$$
\bar{e}=\arg \max _{e \in R_{++}^{N}}\{W(e)-\bar{p} e\} .
$$

That is, $(\bar{p}, x(\bar{p}))$, is the observed market demand of the representative agent, where $x(\bar{p})=\bar{e}$ model. If

$$
\frac{1}{\lambda_{i}} g_{i}(x) \equiv U_{i}(x)
$$


then $U_{i}\left(x_{i}\right)$ is quasilinear, then the representative agent's utility function in Bewley's Marshallian general equilibrium model is quasilinear and

$$
\begin{gathered}
W(e)=\max _{\left\{x_{1}, \ldots, x_{I}\right\} \in \mathbb{R}_{++}^{n I}}\left[\sum_{i=1}^{I} U_{i}\left(x_{i}\right)\right] \\
\text { s.t. } \sum_{i=1}^{I} x_{i}=e .
\end{gathered}
$$

$(\bar{p}, x(\bar{p}))$ is an equilibrium of the exchange economy with consumers endowed with $\left\{U_{i}\left(x_{i}\right)\right\}_{i=1}^{I}$ and social endowment $\bar{e}$ iff 's demand function is cyclically monotone iff it is rationalizable with a quasilinear utility function. That is, the representative agent is endowed with a quasilinear utility function $W(e)$.

Corollary 7 The refutable implications of Marshall's cardinal theory of value can be decided in polynomial time

$$
\bar{e}=\arg \max _{e \in R_{++}^{N}}\{W(e)-\bar{p} e\} .
$$

Brown and Calsamiglia show that a consumer.

Proof The Afriat inequalities, where the marginal utilities of income equal 1, is a family of linear inequalities. The unknowns are the utility levels and the consumer's demands in each observation. Hence feasibility can be decided in polynomial time using interior-point methods.

Cherchye et al. (2011) have recently shown that feasibility of the Walrasian equilibrium inequalities reformulated as an integer programming problem is NP-complete. That is, at present there is no known polynomial time method for deciding the feasibility of the reformulated Walrasian equilibrium inequalities. Moreover, it is widely conjectured that no polynomial time decision procedure is possible for the integer programming problem considered by Cherchye et al.

All of our results: existence, uniqueness, optimality, tatonnement stability and refutability extend to the Marshallian general equilibrium model with production. Optimality, tatonnement stability and refutability follow from the well-known duality result in convex analysis that the supply function is the gradient of the profit function or conjugate of the cost function. As such, the supply function is also cyclically monotone. The cyclical monotonicity of aggregate supply and aggregate demand guarantee (i) that producer and consumer surplus are well defined, (ii) that the excess demand function is cyclically monotone and (iii) that the aggregate demand function and the aggregate supply function are refutable. As in Bewley (2007), existence is shown by maximizing the representative agent's utility function over the compact set of feasible production plans. If this set is strictly convex then the optimum is unique and the supporting prices are the equilibrium prices. See Bewley's Chap. 8 on short-run equilibria for detailed proofs of existence, uniqueness, optimality and tatonnement stability. 


\section{References}

Afriat, S.: The construction of a utility function from demand data. Int. Econ. Rev. 8, 66-67 (1967)

Arrow, K., Hahn, F.: General competitive analysis. San Francisco:Holden-Day (1971)

Basu, S.: Algorithms in Real Algebraic Geometry: A Survey. Department of Mathematics, Purdue University, West Lafayette (2011)

Bewley, T.: The permanent income hypothesis and short-run price stability. J. Econ. Theory 23(3) (1980)

Bewley, T.: General Equilibrium, Overlapping Generations Models, and Optimal Growth Theory. Harvard University Press, Harvard (2007)

Brown, D.J., Calsamiglia, C.: The nonparametric approach to applied welfare analysis. Econ. Theory 31, 183-188 (2007)

Brown, D.J., Matzkin, R.L.: Testable restrictions on the equilibrium manifold. Econometrica 64, 1249-1262 (1996)

Cherchye, L., et al.: Testable implications of general equilibrium models: an integer programming approach. J. Math. Econ. 47, 564-575 (2011)

Cournot, A.A.: Researches into the mathematical principles of the theory of wealth, section 22 (1838)

Dupuit, A.A.: On the Measurement of the Utility of Public Works, republished in 1933 as De l'utilite et sa measure (M. de Bernardi ed.). Riforma Sociale, Turin (1844)

Hildenbrand, W.: On the law of demand. Econometrica 51, 997-1019 (1983)

Marshall, A.: Principles of Economics. MacMillan \& Co, London (1890)

Mas-Colell, A., Whinston, M.D., Green, J.R.: Microeconomic Theory. Oxford University Press, Oxford (1995)

Quah, J.: The monotonicity of individual and market demand. Econometrica 68, 911-930 (2000)

Rockafellar, R.T.: Convex Analysis. Princeton University Press, Princeton (1970)

Varian, H.: The nonparametric approach to demand analysis. Econometrica 50, 945-973 (1983)

Walras, L.: Eléments d'èconomie politique pure; ou, théorie de la richesse sociale, 4th edn. Rouge, Lausanne (1900) 